\title{
A Multitexture Model for Multilook Polarimetric Synthetic Aperture Radar Data
}

\author{
Torbjørn Eltoft, Member, IEEE, Stian N. Anfinsen, Member, IEEE, and Anthony P. Doulgeris, Member, IEEE
}

\begin{abstract}
A statistical model for multilook polarimetric radar data is presented where the polarimetric channels are associated with individual texture variables having potentially different statistical properties. The feasibility of producing closed form probability density functions under certain restrictions is outlined. Mellin kind statistics are derived under various assumptions on the texture variables, and the potential for model fit assessment and hypothesis testing in the Mellin domain is demonstrated. Application to real data proves the usefulness of the analytic approach.
\end{abstract}

Index Terms-Radar polarimetry, synthetic aperture radar, probability density functions, texture modeling

\section{INTRODUCTION}

$\mathbf{K}$ NOWLEDGE of the exact statistical properties of polarimetric synthetic aperture radar (SAR) data forms the basis for many image analysis techniques such as segmentation and land cover classification (e.g. [1], [2]) or speckle filtering [3]. In the literature, Gaussian signal statistics is often taken as the default hypothesis [4]. Analysis of real SAR images reveals that non-Gaussian models give better representation of the data (e.g. [5]), especially at high resolution. For a single channel, successful models have been constructed by treating the distributed targets as a collection of random scatterers with fluctuations in the number of scatterers within each cell, leading to e.g. the well-established K-distribution [5]. The K-distribution models can alternatively be constructed in the framework of the so-called product model, in which the backscattered signals are modeled as a product between a positive scalar texture component and a complex Gaussian speckle variable (e.g. [6], [7]). This recipe produces non-Gaussian probability density functions (pdfs), in which the characteristic properties like moments and cumulants are determined by the actual distribution selected for texture.

The product model can easily be extended to the multivariate case in order to provide non-Gaussian statistical representation of fully polarimetric SAR data. In the simplest and most common case, the backscattered signal is modeled as the product between a random scalar texture component and a Gaussian vector component, representing speckle. Hence, the assumption is that the texture equally effects all polarimetric channels. This model has been widely used in nonGaussian modeling, processing, and analysis of polarimetric SAR images, applying a number of different distributions for the texture, including the gamma distribution [2], [8], the

The authors are with the Department of Physics and Technology, University of Troms $\varnothing$, NO-9037 Troms $\varnothing$, Norway (e-mail: \{torborn.eltoft; stian.normann.anfinsen; anthony.p.doulgeris\}@uit.no).

Digital Object Identifier XX.XXXX/TGRS.200X.XXXXXX inverse gamma distribution [9], and the Fisher distribution [10], to name a few.

The assumption of an equal texture component in all polarimetric channels has to some extent been disputed by experts in the remote sensing community (e.g. [3], [11]). A study performed by Joughin et al. in [12] concluded that for multilook polarimetric data, the scalar product model appears to be exact or at least, to be a satisfactory approximation. Sheen and Johnson [13], on the other hand, showed experimentally that texture might not be independent on polarimetric channel. One reason why the scalar texture model may not be valid is that different scattering mechanisms will contribute differently to the polarimetric channels. For example, volume scattering will produce a strong cross-polarization component, whereas surface and double bounce scattering will contribute stronger to the co-polarized channels. The volume and surface scattering mechanisms would be expected to have different texture properties because of their physically distinct origins. Hence, in forested areas, where the returned signal will have contributions from surface, double bounce, and volume scattering, the scalar texture model may not be suitable. In [14] the validity of the above model was extensively studied for many vegetation types, using low resolution polarimetric data at $\mathrm{C}, \mathrm{L}$ and $\mathrm{P}$ bands, gathered by the AirSAR system over the Feltwell U.K. agricultural test site. This study verified that C, $\mathrm{L}$ and $\mathrm{P}$ band observations over a range of vegetation types are consistent with the scalar texture model, but that there were some evidence of the modeling breaking down.

A more general multivariate product model would naturally associate an individual texture variable with each polarimetric channel. This model will henceforth be referred to as $a$ multitexture product model. This type of model has only received limited attention in the literature, possibly due to the added technical complexity involved with this extension of the texture. In [15], a generalized polarimetric K-distribution based on a trivariate joint gamma distribution for the texture was theoretically discussed, but without any experimental validation. In [16], the authors propose to apply a similar model in a maximum likelihood speckle reduction filter, reporting promising results.

In this paper, we introduce and examine a polarimetric multitexture product model, which allows each polarimetric channel to be characterized by an individual random texture variable. We derive a closed form expression for the pdf of the multilooked covariance matrix under the assumption of reciprocity and reflection symmetry. Taking advantage of the recently introduced Mellin kind statistics [17]-[19], we also derive general expressions for the associated log-cumulants, 
and discuss how the model parameters can be estimated from data. The validity of the model is then analyzed using sample data sets from a diversity of distributed target surfaces.

The paper is organized as follows: Section III presents the theoretical foundations of the statistical modeling and derives the pdf of the multilooked sample covariance matrix in the case of reflection symmetry and reciprocity; Section IIII outlines the framework of Mellin kind statistics with respect to certain cases of the proposed multitexture model; Section IV] covers log-cumulant parameter estimation and a multitexture hypothesis test; and Section $\mathrm{V}$ describes and performs extensive validation tests using real data. Finally, section VI gives some conclusions.

This paper is an extended version of [20].

\section{FundAMENTAL THEORY}

\section{A. Multitexture product model}

Let the scattering vector be given as

$$
\mathbf{s}=\left[S_{h h} S_{h v} S_{v h} S_{v v}\right]^{T},
$$

where the subscripted indices refer to the transmit and receive polarization, in that order, and $(\cdot)^{T}$ denotes matrix transposition. In the multitexture statistical model we have

$$
\mathbf{s}=\mathbf{T}^{1 / 2} \mathbf{x}
$$

where $\mathbf{T}=\operatorname{diag}\left\{T_{h h}, T_{h v}, T_{v h}, T_{v v}\right\}$ is a diagonal matrix containing positive, scalar texture variables associated with the respective polarimetric channels, $(\cdot)^{1 / 2}$ is the matrix square root operator, and $\mathrm{x}$ is a zero mean, circular complex Gaussian vector variable, representing speckle [15]. Hence, the covariance of $\mathbf{s}$ conditioned on the texture matrix $\mathbf{T}$ is

$$
\boldsymbol{\Sigma}_{\mathbf{s}} \mid \mathbf{T}=\mathbf{T}^{1 / 2} \boldsymbol{\Sigma}_{\mathbf{x}} \mathbf{T}^{1 / 2}
$$

with $\Sigma_{\mathrm{x}}$ being the covariance of $\mathrm{x}$. It is well-known that the probability density function (pdf) of the sample covariance matrix for $\mathrm{x}$ follows the scaled complex Wishart distribution [21], which is a slightly modified version of the standard complex Wishart introduced in [22]. Let this sample covariance matrix be given by

$$
\mathbf{W}=\frac{1}{L} \sum_{i=1}^{L} \mathbf{x}_{i} \mathbf{x}_{i}^{H}
$$

where $(\cdot)^{H}$ denotes the Hermitian transpose, and $\mathbf{W} \in \Omega_{+}$, which is the space of positive definite Hermitian matrices. Its pdf becomes

$$
f_{\mathbf{W}}\left(\mathbf{W} ; L, \boldsymbol{\Sigma}_{\mathbf{x}}\right)=\frac{L^{L d}}{\Gamma_{d}(L)} \frac{|\mathbf{W}|^{L-d}}{\left|\boldsymbol{\Sigma}_{\mathbf{x}}\right|^{L}} \operatorname{etr}\left(-L \boldsymbol{\Sigma}_{\mathbf{x}}^{-1} \mathbf{W}\right)
$$

where the vector dimension $d=\operatorname{dim}(\mathbf{x})=4$ for fullpolarimetric SAR data, $|\cdot|$ is the determinant, $\operatorname{etr}(\cdot)=$ $\exp (\operatorname{tr}(\cdot))$ is the exponential trace operator, and $\Gamma_{d}(\cdot)$ is the multivariate gamma function of the complex kind [19]. Furthermore, if texture can be assumed constant on the scale of the multilook window, the sample covariance matrix of $\mathbf{s}$ becomes

$$
\mathbf{C}=\frac{1}{L} \sum_{i=1}^{L} \mathbf{s}_{i} \mathbf{s}_{i}^{H}=\mathbf{T}^{1 / 2} \mathbf{W} \mathbf{T}^{1 / 2} .
$$

Hence, the pdf of $\mathbf{C} \in \Omega_{+}$, given $\mathbf{T}$, is readily obtained from (5) as

$$
\begin{aligned}
& f_{\mathbf{C} \mid \mathbf{T}}\left(\mathbf{C} \mid \mathbf{T} ; L, \boldsymbol{\Sigma}_{\mathbf{x}}\right) \\
& \quad=f_{\mathbf{W}}\left(\mathbf{T}^{-1 / 2} \mathbf{C} \mathbf{T}^{-1 / 2} ; L, \boldsymbol{\Sigma}_{\mathbf{x}}\right) \cdot\left|J_{\mathbf{W} \rightarrow \mathbf{C}}\right| \\
& \quad=\frac{L^{L d}}{\Gamma_{d}(L)} \frac{|\mathbf{C}|^{L-d}}{|\mathbf{T}|^{L}\left|\boldsymbol{\Sigma}_{\mathbf{x}}\right|^{L}} \operatorname{etr}\left(-L \boldsymbol{\Sigma}_{\mathbf{x}}^{-1} \mathbf{T}^{-1 / 2} \mathbf{C} \mathbf{T}^{-1 / 2}\right)
\end{aligned}
$$

where $J_{\mathbf{W} \rightarrow \mathbf{C}}=L^{L d}$ is the Jacobian of the transformation from $\mathbf{W}$ to $\mathbf{C}$. The marginal distribution for $\mathbf{C}$ is obtained by integrating over the pdf of $\mathbf{T}$, i.e.,

$$
f_{\mathbf{C}}\left(\mathbf{C} ; L, \boldsymbol{\Sigma}_{\mathbf{x}}\right)=\int f_{\mathbf{C} \mid \mathbf{T}}\left(\mathbf{C} \mid \mathbf{T} ; L, \boldsymbol{\Sigma}_{\mathbf{x}}\right) f_{\mathbf{T}}(\mathbf{T}) d \mathbf{T} .
$$

\section{B. Reflection Symmetry}

The four-by-four covariance matrix obtained when $d=4$ gives a complete description of the polarimetric backscattering properties of a target. With $\mathrm{s}$ as in (1), we have

$$
\boldsymbol{\Sigma}_{\mathbf{s}}=\mathrm{E}\left\{\mathbf{s s}^{H}\right\}=\left[\begin{array}{llll}
\sigma_{h h h h} & \sigma_{h h h v} & \sigma_{h h v h} & \sigma_{h h v v} \\
\sigma_{h h h v}^{*} & \sigma_{h v h v} & \sigma_{h v v h} & \sigma_{h v v v} \\
\sigma_{h h v h}^{*} & \sigma_{h v v h}^{*} & \sigma_{v h v h} & \sigma_{v h v v} \\
\sigma_{h h v v}^{*} & \sigma_{h v v v}^{*} & \sigma_{v h v v}^{*} & \sigma_{v v v v}^{*}
\end{array}\right] .
$$

If we assume reflection symmetry [23], [24], then (9) simplifies to

$$
\boldsymbol{\Sigma}_{\mathbf{s}}=\left[\begin{array}{cccc}
\sigma_{h h h h} & 0 & 0 & \sigma_{h h v v} \\
0 & \sigma_{h v h v} & \sigma_{h v v h} & 0 \\
0 & \sigma_{h v v h}^{*} & \sigma_{v h v h} & 0 \\
\sigma_{h h v v}^{*} & 0 & 0 & \sigma_{v v v v}
\end{array}\right] .
$$

It is noted that $\boldsymbol{\Sigma}_{\mathbf{S}}=\mathrm{E}_{\mathbf{T}}\left\{\mathbf{T}^{\frac{1}{2}} \boldsymbol{\Sigma}_{\mathbf{x}} \mathbf{T}^{\frac{1}{2}}\right\}$, where $\mathrm{E}_{\mathbf{T}}\{\cdot\}$ denotes expectation with regard to the texture matrix variable $\mathbf{T}$, and $\boldsymbol{\Sigma}_{\mathbf{x}}$ has the same structure as $\boldsymbol{\Sigma}_{\mathbf{s}}$ with regard to zero elements. It is easily shown that the inverse matrix $\Sigma_{\mathbf{x}}^{-1}$ will also have the same zero elements as $\boldsymbol{\Sigma}_{\mathbf{x}}$. Let $q_{i, j}$ and $c_{i, j}$ denote entry $(i, j)$ of $\boldsymbol{\Sigma}_{\mathbf{x}}^{-1}$ and the sample covariance matrix C, respectively. Eq. (7) then takes the form

$$
\begin{array}{r}
f_{\mathbf{C} \mid \mathbf{T}}\left(\mathbf{C} \mid \mathbf{T} ; L, \boldsymbol{\Sigma}_{\mathbf{x}}\right)=\frac{L^{4 L}}{\Gamma_{4}(L)} \frac{|\mathbf{C}|^{L-4}}{\left|\boldsymbol{\Sigma}_{\mathbf{x}}\right|^{L}} \frac{1}{T_{h h} T_{h v} T_{v h} T_{v v}} \\
\times \exp \left\{-L\left(\frac{q_{11} c_{11}}{T_{h h}}+\frac{q_{14} c_{41}+q_{41} c_{14}}{\sqrt{T_{h h} T_{v v}}}+\frac{q_{44} c_{44}}{T_{h v}} .\right.\right. \\
\left.\left.+\frac{q_{22} c_{22}}{T_{h v}}+\frac{q_{23} c_{32}+q_{32} c_{23}}{\sqrt{T_{h v} T_{v h}}}+\frac{q_{33} c_{33}}{T_{v h}}\right)\right\}
\end{array}
$$

We further make the assumption that the texture components $T_{h h}$ and $T_{v v}$ are fully correlated and can be represented by a single random variable, which we shall call $T_{c o}$. Likewise, $T_{h v}$ and $T_{v h}$ are assumed to be fully correlated, and can thus be represented by $T_{x}$. The distribution of the sample covariance matrix $\mathbf{C}$ under the assumption of monostatic radar geometry 
and reflection symmetry then becomes

$$
\begin{aligned}
& f_{\mathbf{C}}\left(\mathbf{C} ; L, \boldsymbol{\Sigma}_{\mathbf{x}}, \boldsymbol{\theta}\right)=\frac{L^{4 L}}{\Gamma_{4}(L)} \frac{|\mathbf{C}|^{L-4}}{\left|\boldsymbol{\Sigma}_{\mathbf{x}}\right|^{L}} \\
& \times \int \exp \left\{-L\left(\frac{q_{11} c_{11}+q_{14} c_{41}+q_{41} c_{14}+q_{44} c_{44}}{T_{c o}}\right)\right\} \\
& \times \frac{f_{T_{c o}}\left(T_{c o} ; \boldsymbol{\theta}_{c o}\right)}{T_{c o}^{2 L}} d T_{c o} \\
& \times \int \exp \left\{-L\left(\frac{q_{22} c_{22}+q_{23} c_{32}+q_{32} c_{23}+q_{33} c_{33}}{T_{x}}\right)\right\} \\
& \times \frac{f_{T_{x}}\left(T_{x} ; \boldsymbol{\theta}_{x}\right)}{T_{x}^{2 L}} d T_{x}
\end{aligned}
$$

where $f_{T_{c o}}\left(T_{c o} ; \boldsymbol{\theta}_{c o}\right)$ and $f_{T_{x}}\left(T_{x} ; \boldsymbol{\theta}_{x}\right)$ denote the pdfs of $T_{c o}$ and $T_{x}$, with respective texture parameter vectors, $\boldsymbol{\theta}_{c o}$ and $\boldsymbol{\theta}_{x}$, gathered in $\boldsymbol{\theta}=\left[\boldsymbol{\theta}_{c o}^{T} ; \boldsymbol{\theta}_{x}^{T}\right]^{T}$. The model proposed in Eq. (12) gives the freedom to assume different texture distributions for the co- and cross-polarimetric channels. The integrals can be evaluated in closed form for all recently introduced texture models such as the gamma, inverse gamma, inverse Gaussian, and Fisher distributions.

\section{Reciprocity}

For reciprocal media, the relation $S_{h v}=S_{y h}$ reduces the scattering vector to $\mathbf{s}=\left[S_{h h} \sqrt{2} S_{h v} S_{v v}\right]^{T}$ and the covariance matrix to a three-by-three matrix of the form

$$
\boldsymbol{\Sigma}_{\mathbf{s}}=\left[\begin{array}{ccc}
\sigma_{h h h h} & \sqrt{2} \sigma_{h h h v} & \sigma_{h h v v} \\
\sqrt{2} \sigma_{h h h v}^{*} & 2 \sigma_{h v h v} & \sqrt{2} \sigma_{h v v v} \\
\sigma_{h h v v}^{*} & \sqrt{2} \sigma_{h v v v}^{*} & \sigma_{v v v v}
\end{array}\right]
$$

where the factor $\sqrt{2}$ ensures matrix span invariance. Hence, the multitexture is represented by a three-by-three diagonal matrix, with $\mathbf{T}=\operatorname{diag}\left\{T_{h h}, T_{x}, T_{v v}\right\}$. If we also assume reflection symmetry, then (13) simplifies to

$$
\boldsymbol{\Sigma}_{\mathbf{s}}=\left[\begin{array}{ccc}
\sigma_{h h h h} & 0 & \sigma_{h h v v} \\
0 & 2 \sigma_{h v h v} & 0 \\
\sigma_{h h v v}^{*} & 0 & \sigma_{v v v v}
\end{array}\right]
$$

where again, it is noted that $\boldsymbol{\Sigma}_{\mathbf{x}}$ and $\boldsymbol{\Sigma}_{\mathbf{x}}^{-1}$ have the same zero elements as $\boldsymbol{\Sigma}_{\mathbf{s}}$. Let $q_{i, j}$ and $c_{i, j}$ still denote entry $(i, j)$ of $\boldsymbol{\Sigma}_{\mathbf{x}}^{-1}$ and $\mathbf{C}$, respectively, now defined under reciprocity such that $d=3$. Eq. (7) then takes the form

$$
\begin{array}{r}
f_{\mathbf{C} \mid \mathbf{T}}\left(\mathbf{C} \mid \mathbf{T} ; L, \boldsymbol{\Sigma}_{\mathbf{x}}\right)=\frac{L^{3 L}}{\Gamma_{3}(L)} \frac{|\mathbf{C}|^{L-3}}{\left|\boldsymbol{\Sigma}_{\mathbf{x}}\right|^{L}} \frac{1}{T_{h h} T_{x} T_{v v}} \\
\times \exp \left\{-L\left(\frac{q_{11} c_{11}}{T_{h h}}+\frac{q_{13} c_{31}}{\sqrt{T_{h h} T_{v v}}}+\frac{q_{22} c_{22}}{T_{x}}\right.\right. \\
\left.\left.+\frac{q_{31} c_{13}}{\sqrt{T_{h h} T_{v v}}}+\frac{q_{33} c_{33}}{T_{v v}}\right)\right\}
\end{array}
$$

When we assume that $T_{h h}$ and $T_{v v}$ are fully correlated and can be represented by $T_{c o}$, the distribution of $\mathbf{C}$ under the assumption of monostatic radar geometry, reciprocity and reflection symmetry becomes

$$
\begin{aligned}
& f_{\mathbf{C}}\left(\mathbf{C} ; L, \boldsymbol{\Sigma}_{\mathbf{x}}, \boldsymbol{\theta}\right)=\frac{L^{3 L}}{\Gamma_{3}(L)} \frac{|\mathbf{C}|^{L-3}}{\left|\boldsymbol{\Sigma}_{\mathbf{x}}\right|^{L}} \\
& \times \int \exp \left\{-L\left(\frac{q_{11} c_{11}+q_{13} c_{31}+q_{31} c_{13}+q_{33} c_{33}}{T_{c o}}\right)\right\} \\
& \quad \times \frac{f_{T_{c o}}\left(T_{c o} ; \boldsymbol{\theta}_{c o}\right)}{T_{c o}^{2 L}} d T_{c o} \\
& \times \int \exp \left\{-L\left(\frac{q_{22} c_{22}}{T_{x}}\right)\right\} \frac{f_{T_{x}}\left(T_{x} ; \boldsymbol{\theta}_{x}\right)}{T_{x}^{L}} d T_{x}
\end{aligned}
$$

which produces a closed form expression for specific texture distributions.

It is noted that both integrals in each of (12) and (16) are of the form $I=\int_{0}^{\infty} \exp (-L \eta / t) / t^{\beta} f_{T}(t ; \boldsymbol{\theta}) d t$, where $f_{T}(t ; \boldsymbol{\theta})$ denotes the respective parametric texture distribution, and $\eta$ and $\beta$ are appropriately defined constants. Table I summarizes analytic expressions for this integral corresponding to commonly used texture models in SAR polarimetry. In Table I the symbols $\mathcal{K}, \mathcal{B}$, and $\mathcal{U}$ refer to the modified Bessel function of the second kind, the Beta function, and the Kummer-U functions, receptively. The complete pdf is therefore obtained by putting the tabulated expressions for the integral terms, representing the co-polarized and the cross-polarized channels, into equations (12) or (16). Note that any combination is allowed, giving rise to in total 6 possible solutions for the pdf.

\section{Mellin Kind Statistics}

The Mellin kind statistics (MKS) of the multitexture product model are derived in this section. MKS is founded on the Mellin transform (see e.g. [25]), and was proposed as a theoretical framework for statistical analysis of single polarization amplitude and intensity radar data by Nicolas [17], and extended to multilook polarimetric radar data in [18], [19], [21].

In the context of this paper MKS theory is used to illustrate how multitexture affects the matrix log-cumulants (MLCs), which are efficient measures for parameter estimation and model evaluation.

The definition of the complex matrix-variate Mellin Transform of the pdf $f_{\mathbf{C}}\left(\mathbf{C} ; L, \boldsymbol{\Sigma}_{\mathbf{x}}, \boldsymbol{\theta}\right)[21$ is

$$
\begin{aligned}
& \mathcal{M}\left\{f_{\mathbf{C}}\left(\mathbf{C} ; L, \boldsymbol{\Sigma}_{\mathbf{x}}, \boldsymbol{\theta}\right)\right\}(s) \\
& =\int_{\Omega_{+}}|\mathbf{C}|^{s-d} f_{\mathbf{C}}\left(\mathbf{C} ; L, \boldsymbol{\Sigma}_{\mathbf{x}}, \boldsymbol{\theta}\right) d \mathbf{C},
\end{aligned}
$$

where $s \in \mathbb{C}$. The resulting complex function is known as the Mellin kind characteristic function (cf),

$$
\phi_{\mathbf{C}}(s)=\mathcal{M}\left\{f_{\mathbf{C}}(\mathbf{C})\right\}(s)=\mathrm{E}\left\{|\mathbf{C}|^{s-d}\right\},
$$

and its logarithm is the Mellin kind cumulant generating function,

$$
\varphi_{\mathbf{C}}(s)=\ln \phi_{\mathbf{C}}(s) .
$$

Matrix log-moments (MLM), $\mu_{\nu}\{\mathbf{C}\}$, and matrix $\log$ cumulants, $\kappa_{\nu}\{\mathbf{C}\}$, are generated by taking the derivatives of $\phi(s)$ and $\varphi(s)$, respectively, evaluated at $s=d$. For example,

$$
\kappa_{\nu}\{\mathbf{C}\}=\left.\frac{d^{\nu}}{d s^{\nu}} \varphi_{\mathbf{C}}(s)\right|_{s=d},
$$


TABLE I

ANALYTIC EXPRESSIONS FOR THE INTEGRALS IN 12 AND 16 CORRESPONDING TO THE COMMON TEXTURE MODELS.

\begin{tabular}{|l|l|l|}
\hline Model & Texture pdf, $f_{T}(t ; \boldsymbol{\theta})$ & Integral $I=\int_{0}^{\infty} \frac{\exp (-L \eta / t)}{t^{\beta}} f_{T}(t ; \boldsymbol{\theta}) d t$ \\
\hline Gamma & $f_{T}(t ; \alpha)=\frac{\alpha^{\alpha}}{\Gamma(\alpha)} t^{\alpha-1} \exp (-\alpha t)$ & $I=\frac{2 \alpha^{\alpha}}{\Gamma(\alpha)}\left(\frac{L \eta}{\alpha}\right)\left(\frac{\alpha-\beta}{2}\right) \mathcal{K}_{\alpha-\beta}(2 \sqrt{\alpha L \eta})$ \\
\hline Inverse Gamma & $f_{T}(t ; \lambda)=\frac{(\lambda-1)^{\lambda}}{\Gamma(\lambda)} t^{-(\lambda+1)} \exp \left(-\frac{\lambda-1}{t}\right)$ & $I=\frac{(\lambda-1)^{\lambda}}{\Gamma(\lambda)} \frac{\Gamma(\lambda+\beta)}{(\lambda+L \eta-1)^{\lambda+\beta}}$ \\
\hline Fisher & $f_{T}(t ; \alpha, \lambda)=\mathcal{B}(\alpha, \lambda) \frac{\alpha}{\lambda-1} \frac{\left(\frac{\alpha \tau}{\lambda-1}\right)^{\alpha-1}}{\left(\frac{\alpha \tau}{\lambda-1}+1\right)^{\alpha+\lambda}}$ & $I=\mathcal{B}(\alpha, \lambda) \Gamma(\beta+\lambda)\left(\frac{\alpha}{(\lambda-1)}\right)^{\beta} \mathcal{U}\left(\beta+\lambda, \beta-\alpha+1, \frac{\alpha L \eta}{\lambda-1}\right)$ \\
\hline
\end{tabular}

can be used to retrieve the population MLCs, for your chosen model.

By definition, the matrix log-moment of order $\nu$ is defined as the logarithmic moment of the matrix determinant, i.e.,

$$
\mu_{\nu}\{\mathbf{C}\}=\mathrm{E}\left\{(\log |\mathbf{C}|)^{\nu}\right\} .
$$

Therefore, the sample MLMs of order $\nu$ are computed from data as

$$
\left\langle\mu_{\nu}\{\mathbf{C}\}\right\rangle=\frac{1}{n} \sum_{i=1}^{n}\left(\log |\mathbf{C}|_{i}\right)^{\nu},
$$

and can be combined into the sample MLCs, denoted $\left\langle\kappa_{\nu}\{\mathbf{C}\}\right\rangle$, using the well-known relations between moments and cumulants [26, ch.3], e.g.,

$$
\begin{aligned}
& \kappa_{1}=\mu_{1} \\
& \kappa_{2}=\mu_{2}-\mu_{1}^{2} \\
& \kappa_{3}=\mu_{3}-3 \mu_{2} \mu_{1}+2 \mu_{1}^{3} \\
& \kappa_{4}=\mu_{4}-4 \mu_{3} \mu_{1}-3 \mu_{2}^{2}+12 \mu_{2} \mu_{1}^{2}-6 \mu_{1}^{4} .
\end{aligned}
$$

The reference to the matrix $\mathbf{C}$ is dropped in the above equations since the relations are general and valid for moments and cumulants of all sorts of random variates. Alternatively, the sample MLCs may be calculated from the data power sums using the unique symmetric unbiased estimators known as $k$ statistics by relations given in [26, ch.12].

\section{A. Mulittexture matrix log-cumulants}

Recall that the sample covariance matrix of the multitexture model from (6) is $\mathbf{C}=\mathbf{T}^{\frac{1}{2}} \mathbf{W} \mathbf{T}^{\frac{1}{2}}$. For the matrix product above, it is known that the Mellin kind cf becomes [21]

$$
\phi_{\mathbf{C}}(s)=\phi_{\mathbf{T}}(s) \cdot \phi_{\mathbf{W}}(s),
$$

which shows that the Mellin kind cf decomposes into a product of the texture and the speckle contributions. The log-cumulants then separate, additively, into

$$
\kappa_{\nu}\{\mathbf{C}\}=\kappa_{\nu}\{\mathbf{W}\}+\kappa_{\nu}\{\mathbf{T}\} .
$$

The contribution of the scaled Wishart matrix, $\mathbf{W}$, was derived in [21] as

$$
\phi_{\mathbf{W}}(s)=\frac{\Gamma_{d}(L+s-d)}{\Gamma_{d}(L)}\left(\frac{|\boldsymbol{\Sigma}|}{L^{d}}\right)^{s-d} .
$$

The $\nu$ th-order MLC expressions for the scaled Wishart distribution are therefore given by

$$
\kappa_{\nu}\{\mathbf{W}\}= \begin{cases}\psi_{d}^{(0)}(L)+\ln |\boldsymbol{\Sigma}|-d \ln (L) & , \nu=1 \\ \psi_{d}^{(\nu-1)}(L) & , \nu>1\end{cases}
$$

where $\psi_{d}^{(\nu)}(\cdot)$ is the multivariate polygamma function, defined in [21], $L$ is the effective number of looks, and $d$ is the matrix dimension. The number of looks is estimated for the data set in advance using MLCs as proposed in [27].

\section{B. General Multitexture}

By definition, the Mellin kind $\mathrm{cf}$ of $\mathbf{T}$ is

$$
\phi_{\mathbf{T}}(s)=\mathrm{E}\left\{|\mathbf{T}|^{s-d}\right\},
$$

and since $\mathbf{T}$ is diagonal it follows that

$$
\phi_{\mathbf{T}}(s)=\mathrm{E}\left\{\prod_{i=1}^{d} T_{i}^{s-d}\right\} .
$$

This is the general case, before any assumptions have been made about the correlation between the texture variables.

In this case, the MLMs can be written as

$$
\mu_{\nu}\{\mathbf{T}\}=\mathrm{E}\left\{\left(\log \prod_{i=1}^{d} T_{i}\right)^{\nu}\right\}=\mathrm{E}\left\{\left(\sum_{i=1}^{d} \log T_{i}\right)^{\nu}\right\} .
$$

For $\nu=1$ these texture components are separable, but for higher orders of moments there will potentially exist cross-coupled terms of the form $\log \left\{T_{i}\right\}^{a} \log \left\{T_{i}\right\}^{b}$ for all combinations of $a, b<\nu$, whose expectations would need to be evaluated.

\section{Special Cases}

In the following, let $T_{x} \perp T_{y}$ denote that texture variables $T_{x}$ and $T_{y}$ are statistically independent, and let $T_{x} \| T_{y}$ denote that they are identical. The $h h$ and $v v$ channels are referred to as the co-polarization (co-pol) channels and the $h v$ and $v h$ channels as the cross-polarization (cross-pol) channels. Further, let the complete set of polarimetric channels available be denoted by $\mathcal{P}$, the subset of co-pol channels by $\mathcal{P}_{c o}$, and the subset of cross-pol channels by $\mathcal{P}_{x}$. The respective sizes of these sets are denoted $d, d_{c o}$, and $d_{x}$. Now consider the Mellin kind $\mathrm{cf}$ under particular sets of assumptions. 
Case (i): scalar-texture model

Relations: $T_{h h}\left\|T_{h v}\right\| T_{v h} \| T_{v v}$.

This case corresponds to the familiar scalar product model, where the texture variables are identical for all polarizations, denoted as $T$. The Mellin kind of then becomes [21]

$$
\begin{aligned}
\phi_{\mathbf{T}}(s) & =\mathrm{E}\left\{T^{d(s-d)}\right\} \\
& =\phi_{\mathbf{T}}(d(s-d)+1)
\end{aligned}
$$

where the transformation from $\phi_{T}(s)$ to $\phi_{T}(s-d+1)$ in (31) follows from the definition of the univariate Mellin kind $\mathrm{cf}$ as $\phi_{T}(s)=\mathrm{E}\left\{T^{s-1}\right\}$ [17], [21], [28]. The MLCs become

$$
\kappa_{\nu}\{\mathbf{C}\}=\kappa_{\nu}\{\mathbf{W}\}+d^{\nu} \kappa_{\nu}\{T\} .
$$

\section{Case (ii): dual-texture model}

Relations: $T_{h h}\left\|T_{v v}, T_{h v}\right\| T_{v h}, T_{h h} \perp T_{h v}, T_{v v} \perp T_{v h}$. In this case, the co-pol texture variables are identical, the cross-pol texture variables are identical, and the co-pol texture variables are totally decoupled from the cross-pol texture variables due to statistical independence. Therefore, the copol and cross-pol contributions to $\phi_{\mathbf{T}}(s)$ can be separated as a product.

$$
\begin{aligned}
\phi_{\mathbf{T}}(s) & =\mathrm{E}\left\{\prod_{i \in \mathcal{P}_{c o}}^{d_{c o}} T_{i}^{s-d}\right\} \mathrm{E}\left\{\prod_{i \in \mathcal{P}_{x}}^{d_{x}} T_{i}^{s-d}\right\} \\
& =\mathrm{E}\left\{T_{c o}^{d_{c o}(s-d)}\right\} \mathrm{E}\left\{T_{x}^{d_{x}(s-d)}\right\} \\
& =\phi_{T_{c o}}\left(d_{c o}(s-d)+1\right) \cdot \phi_{T_{x}}\left(d_{x}(s-d)+1\right),
\end{aligned}
$$

where the common co-pol texture variable and the common cross-pol texture variable are denoted $T_{c o}$ and $T_{x}$, respectively. This property translates to an additive decomposition of their MLCs.

$$
\kappa_{\nu}\{\mathbf{C}\}=\kappa_{\nu}\{\mathbf{W}\}+d_{c o}^{\nu} \kappa_{\nu}\left\{T_{c o}\right\}+d_{x}^{\nu} \kappa_{\nu}\left\{T_{x}\right\} .
$$

\section{Case (iii): quad-texture model}

Relations: $T_{h h} \perp T_{h v} \perp T_{v h} \perp T_{v v}$.

All texture variables are in this case mutually independent. When the texture variables for all polarizations are statistically independent, 29) simplifies to

$$
\phi_{\mathbf{T}}(s)=\prod_{i \in \mathcal{P}} \mathrm{E}\left\{T_{i}^{s-d}\right\}=\prod_{i \in \mathcal{P}} \phi_{T_{i}}(s-d+1)
$$

where $\mathcal{P}=\{h h, h v, v h, v v\}$ and $d=4$ when full-polarimetric data are recorded. The MLCs in this case evaluate to

$$
\kappa_{\nu}\{\mathbf{C}\}=\kappa_{\nu}\{\mathbf{W}\}+\sum_{i \in \mathcal{P}} \kappa_{\nu}\left\{T_{i}\right\} .
$$

The alternative multitexture models can be summarized as

$$
\kappa_{\nu}\{\mathbf{T}\}= \begin{cases}d^{\nu} \kappa_{\nu}\{T\} & : \text { case (i) } \\ d_{c o}^{\nu} \kappa_{\nu}\left\{T_{c o}\right\}+d_{x}^{\nu} \kappa_{\nu}\left\{T_{x}\right\} & : \text { case (ii) } \\ d d & : \text { case (iii) } \\ \sum_{i=1}^{d} \kappa_{\nu}\left\{T_{i}\right\} & \end{cases}
$$

In order to compare the results obtained in the special cases treated so far, it is useful to isolate $\kappa_{\nu}\{\mathbf{T}\}$. The texture variables are not directly observable, but their contribution to the MLCs can be measured by removing the theoretical speckle contribution through

$$
\left\langle\kappa_{\nu}\{\mathbf{T}\}\right\rangle=\left\langle\kappa_{\nu}\{\mathbf{C}\}\right\rangle-\kappa_{\nu}\{\mathbf{W}\} .
$$

\section{MLC PARAMETER ESTIMATION AND HYPOTHESIS TESTING}

From section $\Pi$ it follows that the distribution of the sample covariance matrix $\mathbf{C}$ is determined by the model chosen for the texture variables. In the following, we shall assume unit-mean Fisher distributed texture variables with the two shape parameters $\alpha$ and $\lambda$. This corresponds to the matrixvariate $\mathrm{U}$ distribution model [10] for $\mathbf{C}$, and submatrices of C. The U model is chosen because it is one of the most flexible models available, and includes other common models, such the scaled complex Wishart distribution, the $K$-Wishart distribution [2], [29] and the matrix-variate $\mathcal{G}^{0}$ distribution [9], as special cases. In the two-dimensional log-cumulant diagram constructed from $\kappa_{3}$ and $\kappa_{2}$ (see Figs. 2-5), the U distribution spans the area bordered by the mentioned models, the $K$ Wishart distribution to the left, the $\mathcal{G}^{0}$ distribution to the right, and the Wishart at their intersection. These are limiting models as one or both of $\alpha$ and $\lambda$ tend to infinity [18].

\section{A. MLC parameter estimation}

The MLCs form a general basis for efficient parameter estimation in the product models. The $\nu$ th-order MLC expressions for normalized Fisher distributed texture are given by

$$
\kappa_{\nu}\{T\}= \begin{cases}\psi^{(0)}(\alpha)-\psi^{(0)}(\lambda)+\ln \left(\frac{\lambda-1}{\alpha}\right) & , \nu=1 \\ \psi^{(\nu-1)}(\alpha)+(-1)^{\nu} \psi^{(\nu-1)}(\lambda) & , \nu>1\end{cases}
$$

where $\psi^{(\nu)}(\cdot)$ is the ordinary polygamma function and $\alpha, \lambda$ are the two texture parameters. The total multitexture model logcumulants can be computed by using the appropriate scaling factors, $d^{\nu}$ from (37), and adding the Wishart log-cumulant component, from (27), or, alternatively, the texture part can be isolated by using (38).

Sample log-cumulants are estimated for the data by (22) and (23), and represent the sample's location in log-cumulant space. Model parameter estimation is then posed as a minimum distance problem in log-cumulant space [21] between the measured sample log-cumulants and the model log-cumulants as nonlinear functions of the texture parameters $(\alpha, \lambda)$. We use the first three log-cumulant orders, since we need at least as many expressions as the number of parameters to be solved.

It is noted that the above described procedure also applies to multitexture models, in which case the appropriate submatrices of the sample covariance matrices must be used in the parameter estimation process.

\section{B. Hypothesis testing}

The MLCs are also suitable for model assessment. Goodness-of-fit testing can be presented as a distance measure in the log-cumulant space, since the sampling distribution of MLCs can be assumed to be asymptotically multivariate Gaussian with a known covariance matrix [30]. The distance measured between the sample and the model derived 


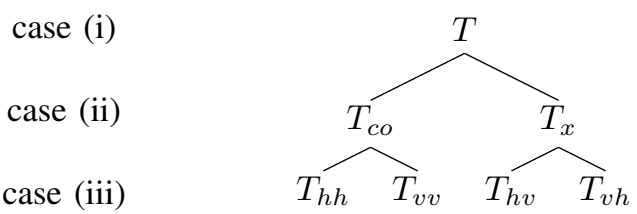

Fig. 1. Hypothesis test scheme to distinguish between scalar, dual, and quad-texture models

log-cumulants in log-cumulant space, hence becomes a chisquared test statistic in hypothesis tests with the number of log-cumulants as the degrees of freedom. That is, three degrees of freedom, since we use the first three log-cumulant orders, $\kappa_{1}, \kappa_{2}, \kappa_{3}$, in our examples. For more details on the goodnessof-fit testing the reader is referred to [30].

The MKS framework provides tools suitable for selection of the appropriate texture model for a particular image data set, for model parameter estimation and for measuring how well the model fits the data. In particular, the goodness-offit (GoF) testing described in [30] can be used to develop as series of multitexture hypothesis tests to determine which multitexture case is most appropriate for a given data set, or for an individual data class.

Three tests are sufficient to distinguish the three cases described in Section III-C and Fig. 1 illustrates how the testing proceeds. First test all four channels combined for a common scalar texture variable, referred to as the scalar-test. If the test passes, we have case (i), the traditional scalar-texture model. Otherwise, test both the co-pol channels for a common texture variable and the cross-pol channels for a common texture variable. If these tests pass, we have case (ii), the dual-texture model. Otherwise, we have case (iii), with independent texture variables for all channels, the quad-texture model.

\section{RESULTS}

The goodness-of-fit tests can be applied on pixel samples from a chosen area, in a sliding window, or on segmented areas or clusters extracted by an algorithm. Our examples are chosen manually and use 529 pixels in a square window. The sample log-cumulants are determined for each of the four channels individually. The hypothesis tests for the scalar and dual-texture cases require the log-cumulants and model parameters of the appropriate common models, and a chosen confidence level, e.g. 95\%. We simply take the mean of the sample texture log-cumulant values for the individual channels to be tested and estimate the Kummer-U model parameters by the minimum distance method explained in Section IV-A. The common scalar-test uses the mean of all four channels, the two dual-texture tests average the two co-pol values and the two cross-pol values, separately. Note that we use the first three log-cumulant orders and set $d=1$ because we have estimated each channel cumulant individually.

Since we use log-cumulants so strongly in these tests, we will first demonstrate their accuracy for parameter estimation. For this we include two simulation experiments: firstly, a visualisation of log-cumulant sensitivity for detecting a distribution's textural variation over several multilook levels; and,
TABLE II

GENERATED AND ESTIMATED MODEL PARAMETERS.

Note that values $>50$ approach the special limiting cases, and, therefore, these Fisher parameters are good estimates.

\begin{tabular}{|l|l|l|}
\hline & \multicolumn{1}{|c|}{ Generated } & \multicolumn{1}{c|}{ Estimated } \\
\hline \multirow{3}{*}{ Class 1 } & $\frac{\text { Co-pol }}{\text { Gamma } \alpha=10}$ & Fisher distribution \\
& $\frac{\text { Cross-pol }}{\text { Inverse Gamma } \lambda=30}$ & $\alpha=42.4, \lambda=217$ \\
& \multicolumn{1}{|c|}{$\lambda=28.8$} \\
\hline
\end{tabular}

secondly, a numerical example of parameter estimation for a specific dual-texture model.

\section{A. Parameter Estimation and Multilook Levels}

To demonstrate that log-cumulants are sensitive to texture even after a high degree of multilook averaging, we include the two plots in Fig. 2. The top plot is for highly textured data such as from an urban land class and is represented by $\mathcal{G}^{0}$ distributed data (texture is inverse gamma distributed) with $\lambda=1.15$. It shows the measured log-cumulant locations and the estimator's $95 \%$ confidence ellipse for each multilook level. The lower plot shows the same multilook degrees but with Gaussian/Wishart generated data to represent homogeneous classes. We used the same 529 pixel window size and one dimension for the estimation to match the results shown for the other experiments. Both figures demonstrate that the measured sample log-cumulants behave exactly as predicted by the theory and that multilook averaging reduces (though not removes) the non-Gaussianity and, hence, degree of texture, as well as the variance of the estimated values. Note that increasing the sample size for the estimation would also directly decrease the variance.

The second demonstration is for parameter estimation under the proposed dual-texture model. For this we have simulated a dual-texture data-set with different texture models for the copol and cross-pol channels. The co-pol is gamma distributed with $\alpha=10$ and the cross-pol is inverse gamma distributed with $\lambda=30$. The dimension is four and the number of looks is eight. The estimation was performed with a Fisher texture distribution that includes both parameters, $\alpha$ and $\lambda$. The numerical values are given in Table II At this number of looks, the asymptotic distributions are approached for values of $\alpha$ and $\lambda$ over about 50 . Therefore, the estimated parameters are clearly in excellent agreement with the simulated parameters. The dual-texture data histograms and log-cumulant diagram is shown in Fig. 3. Each of the co-pol channels are similar and each of the cross-pol channels are similar, yet the co-pol and cross-pol groupings are different and clearly distinguish the gamma (left curve) and inverse gamma (right curve) models. This example was performed as a whole class clustering scenario and achieves a high accuracy and sensitivity because the sample size is 6400 pixels.

\section{B. Hypothesis Tests}

We can visualize the hypothesis testing results in the $\kappa_{3}, \kappa_{2}$ log-cumulant diagram [21] by plotting the sample log- 

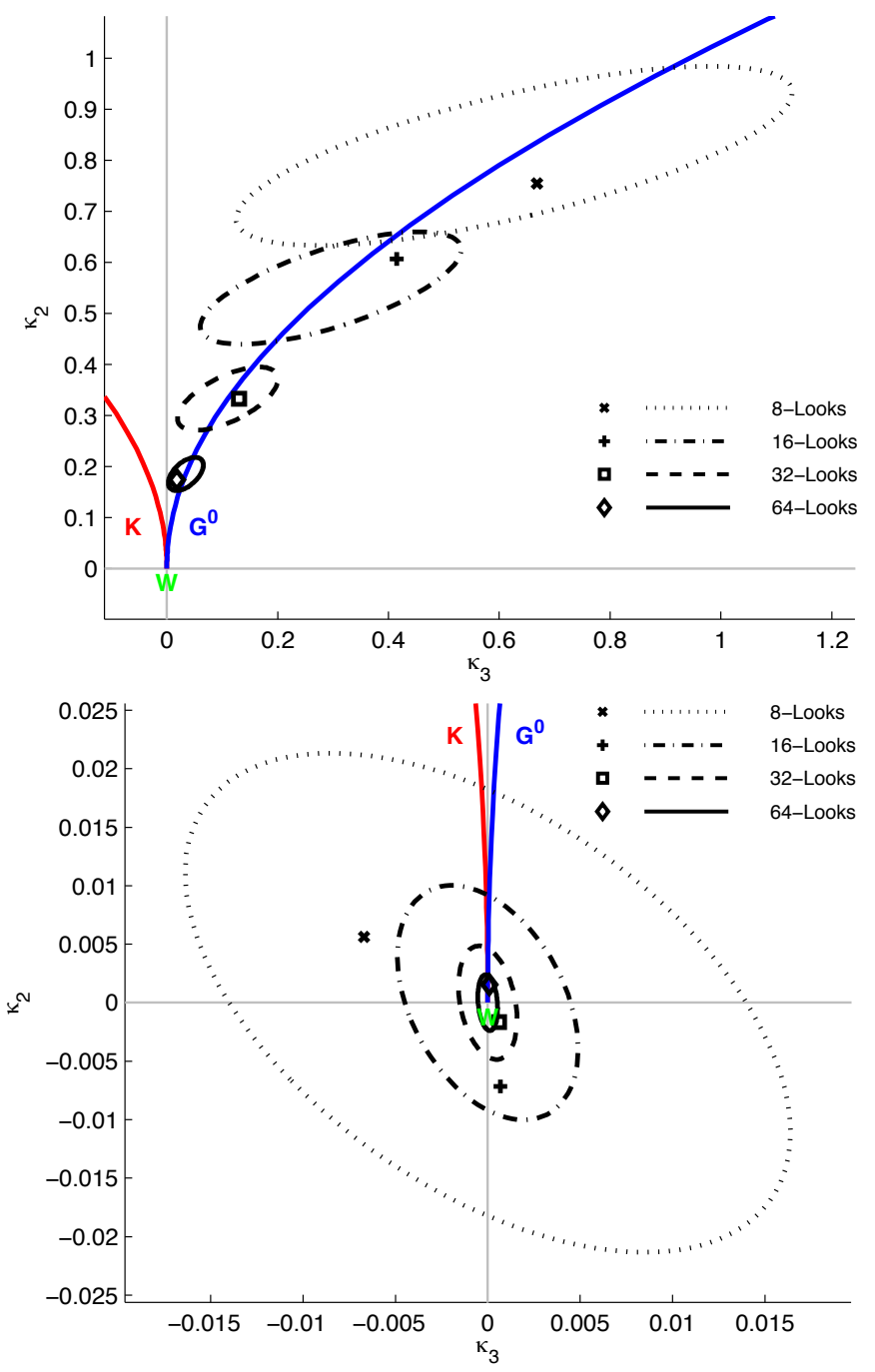

Fig. 2. Multilook level experiment results. The top plot shows the measured sample log-cumulant locations for 8, 16, 32, and 64-look averaging, and the expected $95 \%$ ellipse for the estimator, given highly textured $\mathcal{G}^{0}$ data representing an urban land class. The lower plot shows a similar result for Gaussian/Wishart data expected for homogeneous classes. Both results indicate that the method of log-cumulants is sensitive to detecting texture even after extensive multilooking.

cumulants and the common texture model confidence limit ellipses for each test. In essence, if the appropriate sample points lie within the ellipse, then they pass the test. It is not strictly this simple if more cumulants are used in the testing than these two being displayed, because the true confidence region will be an ellipsoid in all the dimensions. In Figs. 4 to 6. the symbols $\mathrm{HH}, \mathrm{HV}, \mathrm{VH}$ and $\mathrm{VV}$ show the $\left(\kappa_{3}, \kappa_{2}\right)$ points for each individual polarimetric channel. The $95 \%$ confidence ellipses of the common co-pol, cross-pol, and scalar test are shown with dashed, dot-dashed, and solid lines, respectively. The (red) curved line on the left, the (blue) curved line on the right, and the (green) origin where they converge represent population log-cumulants of the $K$ distribution, the $G^{0}$ distribution, and the scaled complex Wishart distribution, respectively.

Fig. 4 presents results for a rain forest sample, from an 8-look L-band PALSAR image of Brasil, where all channels
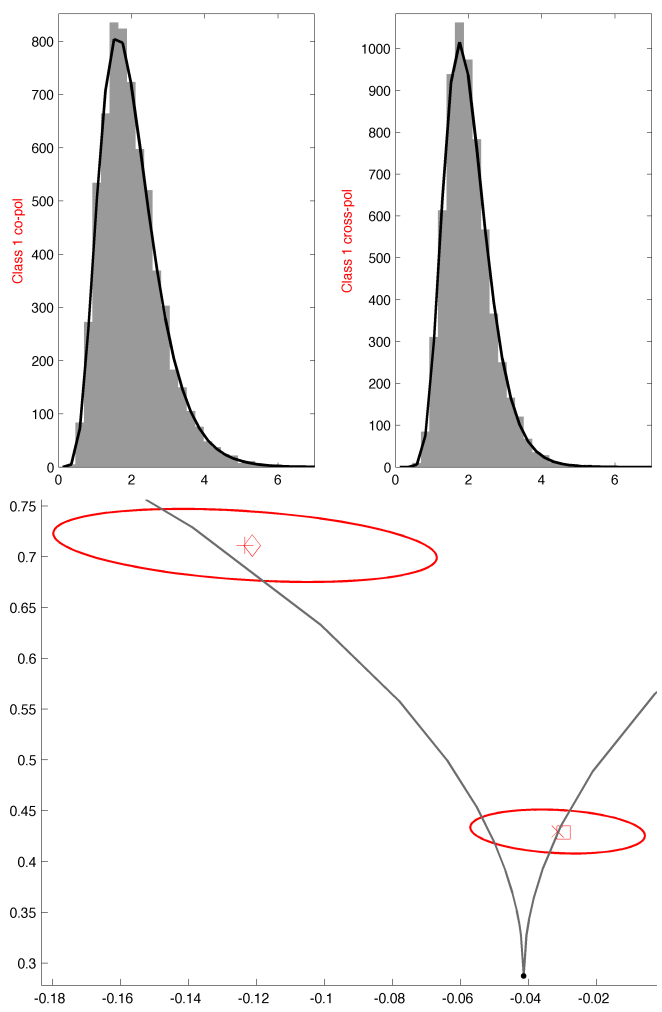

Fig. 3. Dual-texture parameter estimation. The top plot shows the histogram and fitted model to the co-pol and cross-pol data channels. The lower plot shows the estimated log-cumulant locations for the four channels individually, and the expected dual-texture $95 \%$ ellipses. Clearly the model fitting and texture estimation is accurate and the proposed hypothesis testing easily determined that this is a dual-texture model.

fall within the confidence region of the scalar-test (solid line), indicating case (i). This scalar-texture result was by far the most common result for many different thematic regions from many different PolSAR scenes and sensors.

Fig. 5 presents results for a sample of sea ice, from a 32look L-band PALSAR image near Spitsbergen. The scalar-test for the combination of all channels fails, but the co-pol and the cross-pol channels pass the pairwise tests, thereby selecting the multitexture model of case (ii), i.e. dual-texture. Note that this was for a very dark area, possibly grease ice, with very low cross-pol channel intensity.

Fig. 6 presents results for two urban samples from a 25-look C-band Radarsat-2 image of San Francisco. Both show the dual-texture case, but the relative degree of texture is different for the co-pol versus cross-pol channels in both images. Urban samples aligned with the radar have most of the double-bounce scattering mechanism power in the co-pol channels, whereas buildings aligned at $45^{\circ}$ will have high power in the cross-pol channels. The double-bounce mechanism seems to possess the main textural variation, and, hence, the reversed order of the two texture components.

\section{Multitexture mixtures}

While investigating multitexture in different scenes, we observed the significant influence that class mixtures have on texture estimates and the appearance of multitexture. This 

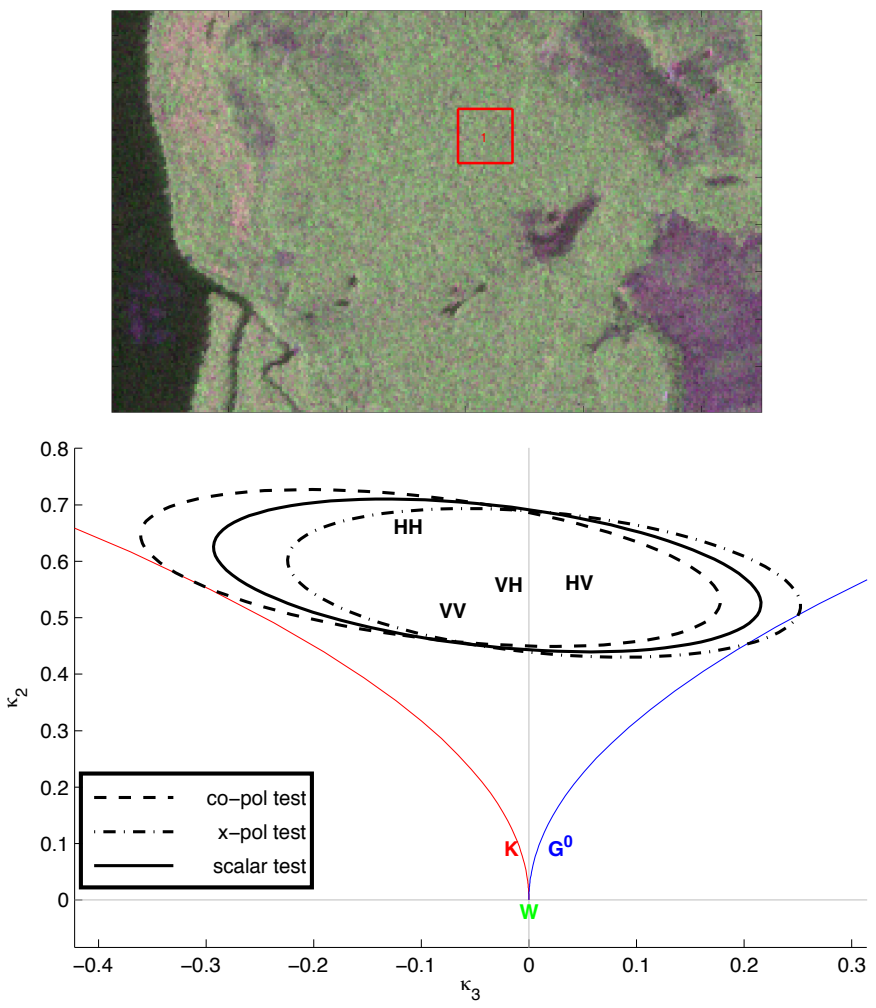

Fig. 4. Pauli RGB image with sample location (top) and Log-cumulant diagram (bottom) with evidence of scalar-texture for a forest segment in a PALSAR image from the Amazon.

is understandable because class mixtures often measure as extreme texture, but it is actually due to mixtures of different brightnesses. One can easily visualize that mixtures with different polarimetry could cause the appearance of multitexture.

Consider two mixture classes with markedly different polarimetry, such that the contrast (relative brightness) between classes is different for the co-pol and cross-pol channels. Fig. 7] shows a two-class mixture example (80:20\% mix) for separate co-pol and cross-pol channels. The dashed (blue) lines represent the two mixture components, the grey histogram is the total mixture distribution and the solid (red) curves are the fitted U distribution models. The solid (red) texture models measured for the co-pol mixture has a different shape than the cross-pol mixture - and thus give the appearance of multitexture. We clearly observed such "mixture multitexture" when we chose a window across a clear class boundary.

It is important to note that such mixtures may be detectable by observing the goodness-of-fit of the histogram to the estimated model, as can be seen by the poorly fitted red curve in the cross-pol channel of fig. 7. However, this may require a very large number of samples to achieve high enough sensitivity. The proposed multitexture model, with channel texture from different origins, would always appear as a continuous texture histogram for each channel, but with different shapes for each channel. The 529 samples used in our examples, Figs. 4 to 6, were not large enough to distinguish such mixed histograms.

Another case for multitexture is for very low intensity
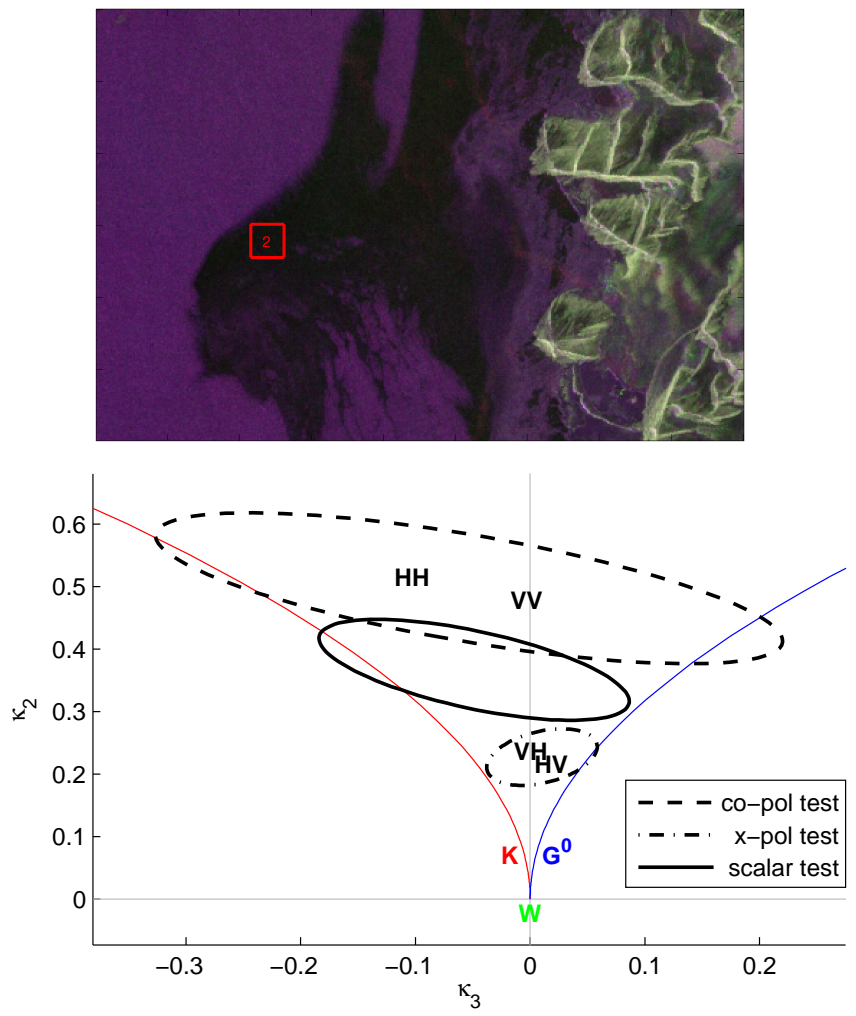

Fig. 5. Pauli RGB image with sample location (top) and Log-cumulant diagram (bottom) with evidence of dual-texture for a sea ice segment in a PALSAR image from Spitsbergen.

channels, such as our sea ice example in Fig. 5 For example over water, the cross-pol channels may be near the noise floor, but the co-pol channels may have a stronger signal. The co-pol characteristics will therefore reflect the multiplicative noise model for speckle, with a particular number of looks and potentially textural variation, but the cross-pol channel could be markedly different. Pure noise in the cross-pol would appear as a narrow Gaussian distribution (appearing as a very high number of looks), while a weak signal near the noisefloor may appear as some sort of mixture and exhibit a high texture. Either case may be measured as a multitexture model.

\section{CONCLUSIONS}

A multitexture model for PolSAR data has been developed, including model density functions, log-cumulant expressions and hypothesis test validation. Preliminary results on several distinctly different PolSAR scenes indicate that the scalar product model is often valid, even for rainforest classes that were suggested to be multitextural due to scattering mechanism arguments. Otherwise, the dual-texture (co-pol/cross-pol paired) case is common.

Care must be taken to avoid class boundaries when sampling images, as the theoretical framework is based upon pure classes and mixtures always result in exaggerated and often multiple textures. This includes the situation of mixtures with the system noise signal if one channel is near the noise-floor. Goodness-of-fit tests may detect such mixed cases, but may require a large sample size to be detectable. This is, perhaps, 

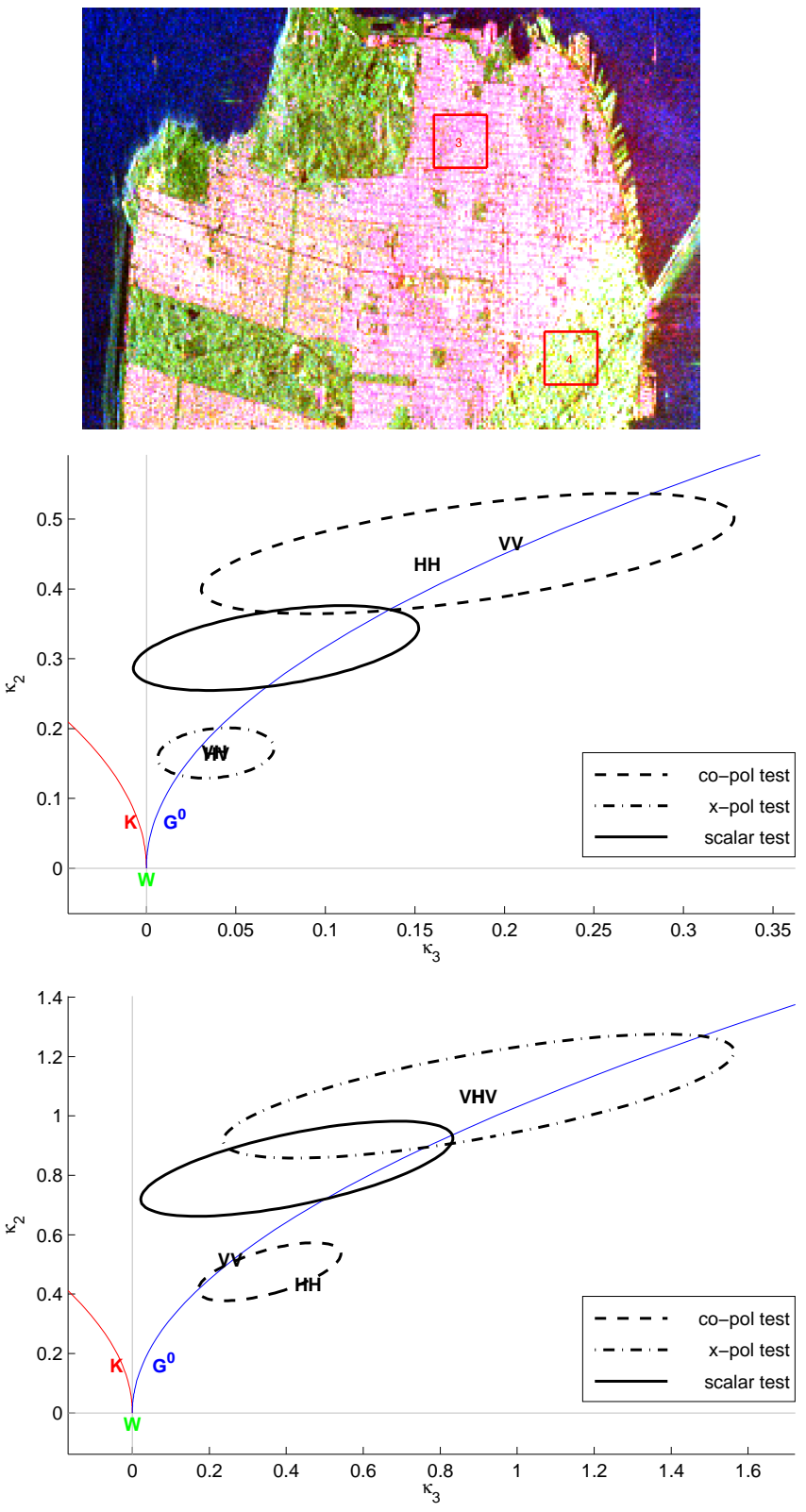

Fig. 6. Pauli RGB image (top) with sample locations for an urban scene of San Francisco in a 25-look C-band Radarsat-2 image. Log-cumulant diagrams for the two boxes depicting buildings aligned with the radar (upper box and plot) and at 45 degrees to radar (lower plot). Both show evidence of dualtexture, but the degree of texture is reversed for the co-pol and cross-pol channels in the two areas.

most suitable to applying tests to entire classes rather than in windows.

In general, it is anticipated that the more representative a statistical model is of the data, the better results will be produced by its application. Hence, if the application method produces any form of measurable textural differences, then a multi-texture approach may achieve better results than scalar texture modeling.

\section{REFERENCES}

[1] J.-M. Beaulieu and R. Touzi, "Segmentation of textured polarimetric sar scenes by likelihood approximation," Geoscience and Remote Sensing, IEEE Transactions on, vol. 42, no. 10, pp. 2063-72, Oct 2004.
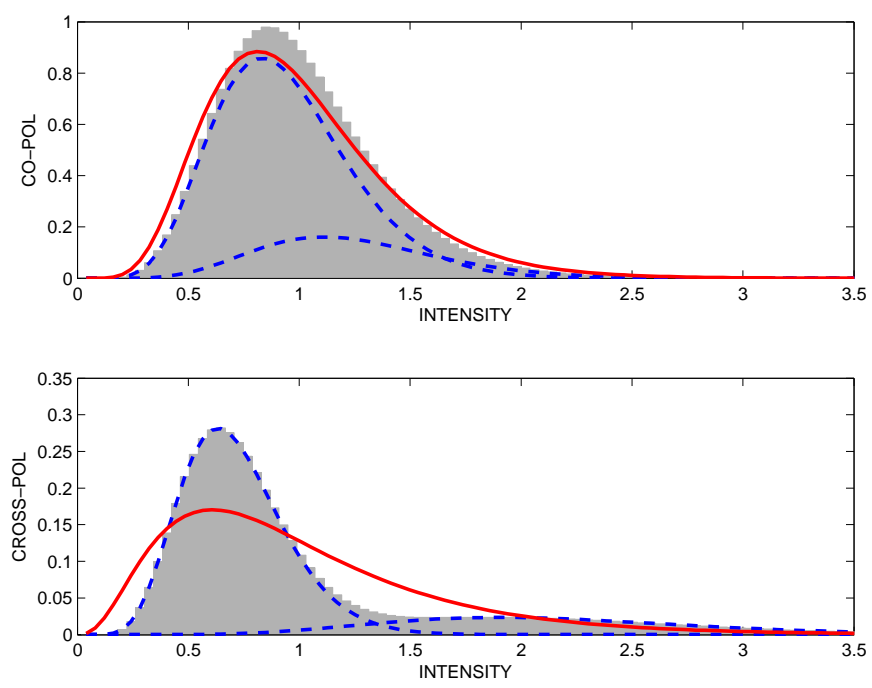

Fig. 7. Multi-texture example of a mixture, showing the co-pol intensity (top) and the cross-pol (bottom). The dashed (blue) curves represent the two mixture components, which give the grey total histogram, with fitted models as solid (red) curves. The different fitted shapes indicate multi-texture.

[2] A. P. Doulgeris, S. N. Anfinsen, and T. Eltoft, "Classification with a non-Gaussian model for PolSAR data," IEEE Trans. Geosci. Remote Sens., vol. 46, no. 10, pp. 2999-3009, Oct. 2008.

[3] A. Lopes and F. Sery, "Optimal speckle reduction for the product model in multilook polarimetric SAR imagery and the Wishart distribution," IEEE Trans. Geosci. Remote Sens., vol. 35, no. 3, pp. 632-647, May 1997.

[4] J. Lee and M. Grunes, "Classification of multi-look polarimetric sar data based on complex wishart distribution," in Telesystems Conference, 1992. NTC-92., National, may 1992, pp. 7/21 -7/24.

[5] E. Jakeman and P. N. Pusey, "A model for non-Rayleigh sea echo," IEEE Trans. Antennas Propag., vol. 24, no. 6, pp. 806-814, Nov. 1976.

[6] J. K. Jao, "Amplitude distribution of composite terrain radar clutter and k-distribbution," IEEE Trans. Antennas Propag., vol. AP-32, no. 10, pp. 1049-1062, Nov. 1984.

[7] T. Eltoft, "The Ricean inverse Gaussian distribution: A new model for non-Rayleigh signal amplitude statistics," IEEE Trans. Image Process., vol. 14, no. 11, pp. 1722-1735, Nov. 2005.

[8] S. H. Yueh, J. A. Kong, J. K. Jao, R. T. Shin, and L. M. Novak, "Kdistribution and polarimetric terrain radar clutter," J. Electrom. Waves Applic., vol. 3, no. 8, pp. 747-768, 1989.

[9] C. C. Freitas, A. C. Frery, and A. H. Correia, "The polarimetric G distribution for SAR data analysis," Environmetrics, vol. 16, no. 1, pp. 13-31, Feb. 2005.

[10] L. Bombrun and J.-M. Beaulieu, "Fisher distribution for texture modeling of polarimetric SAR data," IEEE Geosci. Remote Sens. Lett., vol. 5 , no. 3, pp. 512-516, Jul. 2008.

[11] R. Touzi, W. M. Boerner, J.-S. Lee, and E. Lüneburg, "A review of polarimetry in the context of synthetic aperture radar: Concepts and information extraction," Can. J. Remote Sens., vol. 30, no. 3, pp. 380407, 2004.

[12] I. R. Joughin, D. P. Winebrenner, and D. B. Percival, "Probability density functions for multilook polarimetric signatures," IEEE Trans. Geosci. Remote Sens., vol. 32, no. 3, pp. 562-574, May 1994.

[13] D. Sheen and L. Johnson, "Statistical and spatial properties of forest clutter measured with polarimetric synthetic aperture radar," IEEE Trans. Geosci. Remote Sens., vol. 30, no. 5, pp. 578-88, May 1992.

[14] S. Quegan and I. Rhodes, "Statistical models for polarimetric data: Consequences, testing and validity," Int. J. Remote Sens., vol. 16, no. 7, pp. 1183-1210, May 1995.

[15] Y. Yu, "Textural-partially correlated polarimetric K-distribution," in Proc. IEEE Int. Geosci. Remote Sens. Symp., IGARSS 1998, vol. 4, Seattle, USA, 6-10 July 1998, pp. 2098-2100.

[16] Y. Pi, X. Yang, and G. Liu, "Polarimetric speckle reduction using multitexture maximum likelihood method," Electronic Letters, vol. 39, no. 18, Sep. 2003. 
[17] J.-M. Nicolas, "Introduction aux statistique de deuxième espèce: Application des logs-moments et des logs-cumulants à l'analyse des lois d'images radar," Traitement du Signal, vol. 19, no. 3, pp. 139-167, 2002, in French, English translation in [18].

[18] S. N. Anfinsen, "Statistical analysis of multilook polarimetric radar images with the Mellin transform," Ph.D. dissertation, University of Troms $\varnothing$, Troms $\varnothing$, Norway, May 2010.

[19] S. N. Anfinsen and T. Eltoft, "Analysis of multilook polarimetric radar data with the matrix-variate Mellin transform," in Proc. EUSAR 2010 - 8th Eur. Conf. on Synthetic Aperture Radar, Aachen, Germany, 7-10 Jun. 2010, 4 pp.

[20] T. Eltoft, S. N. Anfinsen, and A. P. Doulgeris, "A multitexture model for multilook polarimetric radar data," in Proc. IEEE Int. Geosci. Remote Sens. Symp., IGARSS 2011, Vancouver, Canada, 24-29 July 2011, pp. 1048-1051.

[21] S. N. Anfinsen and T. Eltoft, "Application of the matrix-variate Mellin transform to analysis of polarimetric radar images," IEEE Trans. Geosci. Remote Sens., vol. 49, no. 6, pp. 2281-2295, Jun. 2011.

[22] N. R. Goodman, "Statistical analysis based on a certain multivariate complex Gaussian distribution (an introduction)," Ann. Math. Statist., vol. 34, no. 1, pp. 152-177, mar 1963.

[23] J.-S. Lee and E. Pottier, Polarimetric Radar Imaging: From Basics to Applications, ser. Optical Science and Engineering. Boca Raton, USA: CRC Press, 2009, no. 143.

[24] S. V. Nghiem, S. H. Yueh, R. Kwok, and F. K. Li, "Symmetry properties in polarimetric remote sensing," Radio Science, vol. 27, no. 5, pp. 693 711, September-October 1992.

[25] P. L. Butzer and J. Stefan, "A self-contained approach to mellin transform analysis for square integrable functions; applications," Integral Transforms and Special Functions, vol. 8, no. 3-4, pp. 175-198, 1999.

[26] A. Stuart and J. K. Ord, Kendall's Advanced Theory of Statistics: Distribution Theory, 6th ed. London, UK: Edward Arnold, 1994, vol. 1.

[27] S. N. Anfinsen, A. P. Doulgeris, and T. Eltoft, "Estimation of the equivalent number of looks in polarimetric SAR imagery," in Proc. IEEE Int. Geosci. Remote Sens. Symp., IGARSS 2008, vol. 4, Boston, USA, Jul. 2008, pp. 487-490.

[28] S. N. Anfinsen, "On the supremacy of logging," in Proc. POLinSAR 2011 - 5th Int. Workshop on Sci. Appl. of SAR Polarimetry and Polarimetric Interferometry, vol. ESA SP-695, Frascati, Italy, 24-28 Jan. 2011, 4 pp.

[29] J. S. Lee, D. L. Schuler, R. H. Lang, and K. J. Ranson, "K-Distribution for multi-look processed polarimetric SAR imagery," in IEEE Int. Geosci. Remote Sensing Symp., vol. 4, Pasedena, USA, August 1994, pp. 2179-2181.

[30] S. N. Anfinsen, A. P. Doulgeris, and T. Eltoft, "Goodness-of-fit tests for multilook polarimetric radar data based on the Mellin transform," IEEE Trans. Geosci. Remote Sens., vol. 49, no. 8, Jul. 2011, 2764-2781.

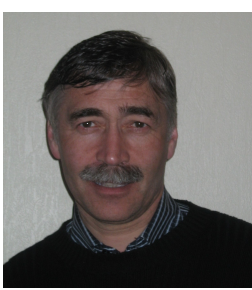

Torbjørn Eltoft (M'92) received the degrees of Cand. Real. (M.S) and Dr. Scient. (Ph.D) from University of Troms $\varnothing$, Norway, in 1981 and 1984, respectively. In 1988 he joined the Faculty of Science and Technology, University of Troms $\emptyset$, where he currently is Head of the Department of Physics and Technology. He is a Professor in electrical engineering, and holds a position as Adjunct Professor at the Northern Research Institute (Norut), Troms $\varnothing$. His research interests include multi-dimensional signal and image analysis with application in radar remote sensing, statistical models, neural networks, and machine learning. Dr. Eltoft has been Associate Editor of the journal Pattern Recognition for five years. He was the recipient of the year 2000 Outstanding Paper Award in Neural Networks awarded by IEEE Neural Networks Council, and of the Honorable Mention for the 2003 Pattern Recognition Journal Best Paper Award.

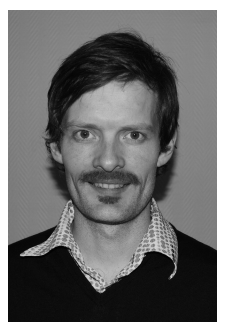

Stian Normann Anfinsen (S'06,M'10) received the Cand. Mag. (B.Sc.), Cand. Scient. (M.Phil.) and $\mathrm{Ph} . \mathrm{D}$. degrees in physics from the University of Tromsø, Troms $\varnothing$, Norway in 1997, 2000 and 2010, respectively. He received the M.Sc. in Communications, Control and Digital Signal Processing with distinction from the University of Strathclyde, Glasgow, U.K. in 1998. He was with satellite ground station system provider Kongsberg Spacetec in Tromsøfrom 2001 to 2005 . He then moved to the University of Troms $\varnothing$, Department of Physics and Technology, where he is currently a postdoctoral research fellow. His current research focuses on statistical modelling, parameter estimation, classification, change detection and target detection in polarimetric synthetic aperture radar images. He has been recognized as the 2012 Best Reviewer of the IEEE Journal of Selected Topics in Applied Earth Observations and Remote Sensing.

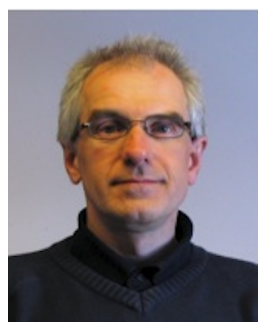

Anthony P. Doulgeris (S'06,M'12) received the B.Sc. degree in physics from The Australian National University, Canberra, Australia, in 1988, the M.Sc. degree and the Ph.D. degree in physics from the Department of Physics and Technology, University of Troms $\varnothing$, Troms $\varnothing$, Norway, in 2006, and 2011, respectively.

$\mathrm{He}$ is continuing his research in applied Earth observation as a postdoctoral research fellow at the Department of Physics and Technology, University of Troms $\varnothing$, Troms $\varnothing$, Norway, and is also affiliated with the Barents Remote Sensing School. His research interests focus on investigating remote sensing, pattern recognition and multidimensional statistical modelling, in particular with polarimetric synthetic aperture radar images. 